\title{
Toxoplasmose et transfusion *
}

\author{
par B. BEAUVAIS, J.-F. GARIN, M. LARIVIERE, G. LANGUILLAT \\ et H. GALAL \\ (Collaboration technique: A. Pougnier et J. Rozier) \\ Laboratoire de Parasitologie-Mycologie ( $\mathrm{P}^{r}$ M. LARIVIÈRE), \\ U.E.R. Lariboisière-Saint-Louis, \\ 15, rue de l'Ecole de Médecine, F 75270 Paris Cedex 06.
}

\section{Résuméé.}

Les toxoplasmes peuvent être transmis par voie transfusionnelle: si la transfusion à partir de donneurs sains fait courir un risque limité aux receveurs, en raison de la parasitémie généralement brève, de l'immunisation rapide du sujet infecté contre son parasite éliminant ainsi la possibilité de rechutes, d'une primoinfection habituellement précoce en France rendant probable l'immunité des donneurs qui sont le plus souvent des adultes, les transfusions, à partir des donneurs de globules blancs atteints de leucémie myéloïde chronique, font courir un risque infiniment plus grand: leurs mécanismes de défenses immunitaires étant altérés, l'apparition au cours de l'évolution de leur maladie d'une primoinfection toxoplasmique est plus fréquente; une reprise évolutive chez des sujets anciennement infectés est possible. En conséquence, la présence des parasites dans le sang de ces malades est plus souvent à craindre.

62 malades atteints le leucémie myélö̈de chronique ont eu des tests sérologiques de la toxoplasmose avant toute chimiothérapie immunodépressive : 7 avaient un titre d'anticorps élevé, avec seulement dans un cas présence d'IgM. Par l'inoculation des leucocytes de 6 d'entre eux à la Souris, la preuve de l'existence d'une parasitémie chez 4 malades a été apportée.

Les donneurs atteints de leucémie myéloïde chronique, à sérologie toxoplasmique élevée même stable et sans $\operatorname{IgM}$, sont donc des donneurs potentiellement dangereux. Une toxoplasmose aiguë chez une petite fille, ayant reçu des leucocytes provenant d'un de ces 4 donneurs, a pu être prouvée d'une manière indiscutable. Il est donc nécessaire d'une part d'éliminer, dans la mesure du possible, ces sujets en tant que donneurs, d'autre part, de poursuivre les investigations en recherchant l'existence d'une parasitémie chez tous les donneurs atteints de leucémie myéloïde chronique quel que soit le taux de leurs anticorps: si elle s'avère fréquente, un traitement par la spiramycine sulfadiazine, qui semble avoir une bonne efficacité sur la parasitémie, devra être donné systématiquement durant quelques jours avant le début des leucophérèses.

(*) Ce travail a fait l'objet d'une communication à la Société Française de Parasitologie, le 17 janvier 1976.

Nous remercions MM. Reviron, Ben Buran et Bussel du Centre de transfusion de l'Hôpital St-Louis de nous avoir ouvert les possibilités de ce travail. 


\section{Summary.}

Toxoplasmosis and Transfusion.

Toxoplasma can be transmitted by transfusion. If recipients incur a limited risk by transfusions from healthy donors, on account of the parasitemia usually short, the fast immunization of the infected subject against his parasite, eliminating thus a possibility of relapse, a primoinfection usually early in France making very likely the donors's immunity as they are mainly adults, recipients incur a much greater risk by transfusions from leucocytes of donors suffering from chronic myeloid leukemia: the immunitary defences of such patients being reduced, a toxoplasmic primoinfection during the disease's evolution is more frequent, a relapse for formely infected subjects is possible. Accordingly, the presence of parasites in such patients's blood is more often to fear.

In 62 patients suffering from chronic myeloid leukemia, antitoxoplasma antibodies were mesured before beginning immuno-suppressive chemotherapy: 7 showed a high antibody title with IgM in one case only. Animal inoculation with leucocytes of 6 from them gave the direct evidence of the parasite's presence in the blood from 4 patients.

Donors suffering from chronic myeloid leukemia, showing a high antibody title, even though it is stable and without IgM are consequently potential «dangerous donors ». A young girl was suffering from an indubitable acute toxoplasmosis, a few time after she had received leucocytes from one of these donors.

So it is absolutely necessary, on the one hand to eliminate, if possible, these subjects as donors, on the other hand to follow up investigations by looking for a parasitemia in every donor suffering from chronic myeloid leukemia whatever serological titles he could have: if the parasitemia is proving frequent, a treatment by Spiramycine-sulfadiazine that seems to be effective opposite parasitemia, will be systematically undertaken before beginning of leucophoresis.

Les toxoplasmes étant des parasites des cellules du système réticulo-histiocytaire, leur présence, dans les monocytes du sang périphérique et éventuellement dans le plasma après éclatement des cellules parasitées, rend possible leur transmission transfusionnelle.

Les transfusions peuvent être réalisées à partir de deux types de donneurs :

- des donneurs «normaux» dont le sang est utilisé soit pour des transfusions du sang total, soit pour des transfusions de leucocytes;

- des donneurs atteints de leucémie myéloïde chronique : qui sont les donneurs de globules blancs les plus «rentables», les leucophérèses permettant d'obtenir chez eux en moyenne 40 fois plus de globules blancs que chez un donneur normal.

\section{I. - Le risque de toxoplasmose transfusionnelle à partir d'un donneur normal :}

Tout en étant réel, en raison de l'extrême diffusion de cette affection parasitaire, il est cependant limité. 
En effet d'une manière générale, chez un sujet normal la parasitémie semble brève et n'exister que dans la période qui suit de près la contamination. Très rapidement, des processus de défenses immunitaires humorales et surtout cellulaires sont élaborés, aboutissant à une destruction des formes libres du toxoplasme. Et lorsque des manifestations cliniques apparaissent (environ 8 à 15 jours après la contamination), si des anticorps antitoxoplasmiques (essentiellement des $\operatorname{IgM}$ à cette date) sont déjà présents dans le sérum, les essais d'isolement du parasite, soit par examen de frottis, soit par inoculation à l'animal, se soldent souvent par un échec.

Ainsi, en dehors de l'éventualité possible d'un sujet ayant une infection toxoplasmique aiguë au moment de sa prise de sang, la présence de toxoplasmes dans le sang d'un donneur est sans doute rare.

On a cependant signalé des cas de parasitémie prolongée : Miller et coll. (1969) en rapporte deux cas (19). L'un concernait une jeune femme qui avait mis au monde un enfant mort-né atteint de toxoplasmose congénitale. Elle n'avait jamais présenté de manifestations cliniques, mais gardait une parasitémie 2, 3 et 14 mois après l'accouchement.

L'autre était celui d'un homme de 26 ans dont la parasitémie fut retrouvée deux mois après la découverte d'une toxoplasmose ganglionnaire, alors que tout signe clinique avait disparu.

Plus récemment Sibalic et coll. (1975) (24) ont pu isoler le toxoplasme par inoculation à la Souris du sang de 4 malades sur 10, 2 mois, 2 ans et 4 ans, après le début de leur maladie.

La pathogénie de cette parasitémie prolongée n'est pas claire et on peut se demander si ces sujets étaient réellement des sujets «normaux » et s'ils ne présentaient pas, en fait, une insuffisance immunitaire. Pourtant, en expérimentation animale, Remington, Melton et Jacobs retrouvaient des toxoplasmes dans le sang de souris, cobayes et lapins jusqu'à un an après l'inoculation d'une souche avirulente. mais ces animaux résistaient à une surinfection par une souche cette fois virulente. Une faillite immunitaire n'était donc sûrement pas la cause de la persistance de la parasitémie.

Quoi qu'il en soit, que la parasitémie soit brève ou plus prolongée, un sujet normal finit toujours par «s'immuniser » contre son parasite, rendant théoriquement impossible une réinfection, une reprise évolutive de la maladie et, de ce fait, une nouvelle parasitémie. En France, la primo-infection toxoplasmique se fait surtout chez le sujet jeune, puisqu'à l'âge de $20-25$ ans, 80 à $85 \%$ de la population possède déjà des anticonps résiduels, témoins d'une infection ancienne. Or les donneurs de sang étant le plus souvent des adultes, ils sont donc, pour la plupart, «immunisés ».

Si néanmoins une parasitémie existe, elle est en général minime. Les transfusions de sang total se font habituellement à partir de flacons stockés à la banque du sang et conservés depuis plus ou moins longtemps à $+4^{\circ}$. Certes le sang humain est un bon milieu de survie pour les toxoplasmes: en effet des parasites provenant d'un exsudat péritonéal de souris infectées 4 jours auparavant et placés dans un tel milieu, survivent 50 jours à $+4^{\circ}$. Mais il n'est pas possible d'extrapoler totalement à partir d'une expérimentation animale qui met en jeu au départ un nombre considérable de 
parasites. Les quelques toxoplasmes éventuellement présents dans le sang doivent voir leurs chances se survie diminuer au fur et à mesure que le stockage à $+4^{\circ}$ se prolonge.

En revanche, les transfusions de leucocytes paraissent offrir un risque supérieur de transmission du parasite en raison des énormes quantités de globules blancs transfusés. Le risque est d'autant plus probable qu'il n'existe pas de possibilités d'altération par le moyen de conservation, le passage du donneur au receveur s'effectuant immédiatement.

En conclusion, la transmission transfusionnelle de toxoplasmes à partir d'un donneur normal est possible, mais certainement rare. Il n'en reste pas moins qu'il ne serait peut-être pas inutile de pratiquer systématiquement chez tous les donneurs, notamment les donneurs étrangers qui sont beaucoup moins souvent immunisés que les Français, une sérologie de la toxoplasmose avec dosage des IgM, afin d'écarter, tout au moins, ceux dont les résultats feraient suspecter une infection récente.

\section{II. - Le risque de transmission transfusionnelle des toxoplasmes à partir des leucocytes de sujets atteints de leucémie myéloüde chronique est infiniment plus grand:}

Comme d'ailleurs tous les malades atteints d'hémopathies malignes en général, ils présentent, du fait de leur maladie elle-même et des thérapeutiques auxquelles ils sont soumis, une défaillance de leur système de défense immunitaire, en particulier cellulaire. Ils sont donc très sensibles à toute agression: les bactéries, les virus, les champignons et les parasites trouvent là un terrain particulièrement favorable à leur intallation et à leur multiplication.

Les toxoplasmes en particulier ne seront pas détruits ou, tout au moins tardivement; ils pourront continuer à se multiplier et envahir tout l'organisme. De plus une rechute ou une reprise évolutive d'une infection ancienne sera possible, soit à la suite d'une réinoculation exogène, soit à la suite d'une rupture kystique avec mise en circulation de nouvelles formes libres.

Ceci rend donc probable chez des sujets atteints de leucémie myéloïde chronique la plus haute prévalence: d'une part d'une infection toxoplasmique évolutive, d'autre part d'une parasitémie. A travers la littérature, et notre expérience personnelle, nous obtenons confirmation de ces hypothèses :

Si l'on peut établir un parallélisme entre un taux d'anticorps élevé et l'existence d'une infection toxoplasmique évolutive ou tout au moins relativement récente, le poucentage de sujets atteints de L.M.C. et présentant cette infection évolutive ou récente est plus grand que celui des sujets normaux :

- Vogel et Lunde (28) utilisant la réaction d'hémagglutination trouvent une proportion de 5 sujets sur 39, soit $12,8 \%$, atteints de L.M.C. avec un titre de $1 / 16000$, tandis que la proportion établie par Kessel dans une population normale d'un groupe d'âge correspondant (au-dessus de 15 ans) n'est que de 5 sur 210 , soit $2,4 \%$; 
- L'un d'entre nous (12) utilisant le test d'agglutination directe des toxoplasmes, a pu faire la même constatation; des titres agglutinants élevés étaient observés dans la proportion de 4 L.M.C. pour un sujet normal ;

- Depuis le début de l'année 1974, nous avons pu suivre sérologiquement 61 malades atteints de leucémie myéloïde chronique. Les réactions utilisées sont les réactions d'agglutination directe de toxoplasmes avant et après réduction du sérum par le 2 Mercapto-éthanol, auxquelles a été adjointe plus récemment la réaction d'immunofluorescence.

7 malades sur les $61(11,5 \%)$ présentaient une sérologie fortement positive:

- avec un titre égal ou supérieur au 1/512 pour 6 d'entre eux, égal au 1/128 pour le $7^{\circ}$, en agglutination directe ;

— un titre supérieur ou égal à 1000 U.I. en immunofluorescence.

Il est important de noter que : aucun de ces malades ne présentait de manifestations cliniques susceptibles d'être rattachées à une toxoplasmose et surtout la recherche d'anticorps antitoxoplasmiques ayant fait partie (comme cela devrait toujours être) du bilan systématique de la leucémie avait été faite avant toute chimiothérapie.

Il nous a paru intéressant de rechercher l'existence d'une parasitémie chez ces malades à sérologie fortement positive et comme ils étaient soumis à des leucophérèses, nous avons pu obtenir des leucocytes de 6 d'entre eux.

Des frottis de ces leucocytes ont été fixés au May Grunwald et colorés au Giemsa. Aucun n'a montré d'éléments parasitaires.

Des inoculations à la Souris par voie intra-péritonéale ont été faites à raison d'1 $\mathrm{ml}$ de suspension leucocytaire par Souris. 5 à 7 semaines plus tard les souris étaient sacrifiées. La positivité de l'inoculation était affirmée par :

la mise en évidence directe du parasite :

— présence de kystes toxoplasmiques à l'examen extemporané des lobes frontaux ;

- apparition d'un exsudat péritonéal avec présence de toxoplasmes au cours du second passage (souris inoculées à partir d'un broyat du cerveau des souris du premier passage) ;

la séro-conversion: avec augmentation progressive du taux des anticorps objectivable en comparant les résultats sérologiques de souris sacrifiées à intervalles réguliers.

ObServation $\mathrm{N}^{\circ}$ 1. - M. M..., 60 ans, atteint de L.M.C. découverte en février 1974. La réaction d'agglutination directe des toxoplasmes, le 11 mars 1974 , donne un titre de I/4096 stable après 2 Mercapto-éthanol. Les anticorps agglutinants sont donc essentiellement des IgG.

Un deuxième prélèvement effectué 10 jours plus tard donne un test sérologique identique au premier.

Le 4 avril, un traitement par Spiramycine Sulfadiazine est commencé et, le même jour, des leucocytes sont inoculés à la Souris : l'inoculation se révélera positive chez 5 souris sur 7 .

Par la suite, de nouveaux essais d'isolement du toxoplasme (mai, novembre, décembre 1974, janvier et février 1975) se sont toujours soldés par un échec. 
Les examens sérologiques régulièrement pratiqués montrent un titre qui ne varie pas. Lo dernier date d'octobre 1975: le titre d'agglutination était toujours au I/4096 stable après 2 Mercapto-éthanol, le titre d'anticorps fluorescents de 2000 U.I. (ce même titre avait été observé en mai 1975).

ObServation $\mathbf{N}^{\circ}$ 2. $-\mathbf{M}^{\mathrm{me}} \mathrm{K} \ldots, 35$ ans, leucémie myélö̈de chronique découverte en août 1974.

La réaction d'agglutination directe des toxoplasmes le 29 août 1974 est positive au I/4096, le titre restant stable après 2 Mercapto-éthanol. Là aussi les anticorps semblent donc essentiellement des IgG. Un traitement par Spiramycine-Sulfadiazine est institué. Le même jour des leucocytes sont inoculés à 5 souris.

L'inoculation donnera un résultat négatif chez les 5 souris.

ObSERVATion $\mathbf{N}^{\circ}$ 3. - M. L..., 61 ans, leucémie myéloïde chronique découverte en août 1974.

La sérologie de la toxoplasmose demandée le 21 août 1974 révèle un titre d'agglutinines totales au I/2048 et un titre d'agglutinines résistantes au 2 Mercapto-éthanol au I/64.

L'indice d'agglutination défini par J.-P. Garin (nombre de tubes d'écart entre le titre des agglutinines totales et des agglutinines résistantes) étant égal à 5, une partie des anticorps semble donc être des IgM.

Le 6 septembre 1974, le malade est mis au traitement par Spiramycine Sulfadiazine et le même jour des leucocytes nous sont adressés.

2 souris sur les 5 inoculées donneront des résultats positifs.

Par la suite de nouveaux essais d'isolement du parasite (novembre, décembre 1974, janvier 1975) sont un échec. Les contrôles réguliers von montrer une augmentation progressive des agglutinines totales et surtout des agglutinines résistantes.

Le 8 janvier 1974: agglutinines totales: $+\mathrm{I} / 4096$; agglutinines résistantes: + I/512.

Le 19 décembre 1974: agglutinines + I/4096, titre stable après 2 Mercapto-éthanol.

Le dernier examen (octobre 1975) montre une baisse du titre agglutinant: + I/512 stable, et un titre de 250 U.I. en immunofluorescence.

ObServation $\mathrm{N}^{\circ}$ 4. $-\mathbf{M}^{\mathrm{me}} \mathrm{T}$..., 39 ans, leucémie myéloïde chronique découverte en octobre 1974.

Le 28 octobre 1974 la réaction d'agglutination directe des toxoplasmes révèle un titre de I/512 sur sérum total et de I/256 après réaction du 2 Mercapto-éthanol. Les anticorps sont donc essentiellement des IgG.

Le traitement par Spiramycine Sulfadiazine est commencé le 4 novembre 1974. Des leucocytes nous sont adressés le même jour.

1 Souris sur les 5 inoculées donne un résultat positif.

Tous les essais d'isolement ultérieur du parasite ont été infructueux (7 novembre, 12 novembre, 15 novembre, décembre 1974, janvier, février, avril 1975).

Les contrôles sérologiques donnent un titre inchangé le 6 novembre 1974, mais montre ensuite une très rapide décroissance, puisque le 4 décembre 1974 , le titre n'est plus qu'au I/16 stable après 2 Mercapto-éthanol. Actuellement le faible titre se maintient tant en agglutination directe qu'en immunofluorescence (50 U.I.).

ObServation $\mathrm{N}^{\circ}$ 5. - $\mathbf{M}^{11 \text { e }}$ B..., 10 ans, leucémie myéloïde chronique découverte en novembre 1974. 
La sérologie toxoplasmique pratiquée à ce moment-là montre un titre d'agglutinines au I/4096 stable après 2 Mercapto-éthanol.

Un traitement par Spiramycine-Sulfadiazine est commencé le 15 novembre 1974. Le même jour et les jours suivants des leucocytes sont inoculés à la Souris : toutes les souris demeureront négatives.

OBSERVATION $\mathrm{N}^{\circ}$ 6. - $\mathrm{M}^{\text {ine }} \mathrm{V}$..., 44 ans, leucémie myélö̈de chronique découverte en octobre 1975 .

La sérologie toxoplasmique faite le 14 octobre montre un titre d'agglutination au $1 / 128$, stable après 2 Mercapto-éthanol, un titre en immunofluorescence égal à 1000 U.I. Des leucocytes sont inoculés à 7 souris le 24 octobre 1975.

Les 7 souris se révéleront positives.

Un traitement à la Spiramycine Sulfadiazine est institué le 27 octobre 1975. Un nouvel essai d'isolement des toxoplasmes est fait à partir des leucocytes du 29 octobre 1975 : 3 souris sur 4 inoculées sont positives.

Mais les inoculations pratiquées à partir des leucocytes du 6 novembre 1975 seront toutes négatives.

4 malades sur les 6 étudiés présentaient donc au moment où leurs leucocytes ont été inoculés une parasitémie.

De ces résultats découlent un certain nombre de considérations:

- En l'absence de références antérieures, il n'est pas possible de dire si cette parasitémie était en rapport avec une primoinfection toxoplasmique ou avec une reprise évolutive de la maladie.

Certes M. L... possédait des IgM lors des premiers examens et les contrôles sérologiques répétés ont montré une ascension de la courbe des anticorps avec disparition progressive de ces IgM. Mais cette ascension du titre s'observe aussi bien au cours d'une rechute que d'une primoinfection, et, bien que rare, une nouvelle synthèse d'IgM spécifiques n'est pas incompatible avec une rechute et a été signalée par plusieurs auteurs.

Si l'on considère l'âge des sujets chez lesquels a été mise en évidence une parasitémie (entre 39 et 60 ans), étant donné la haute incidence de la toxoplasmose en France qui fait que 90 à $95 \%$ des sujets à partir de 40 ans sont porteurs d'anticorps résiduels, la probabilité d'une reprise évolutive est infiniment plus grande que celle d'une primoinfection. Or il est intéressant de le souligner encore, cette reprise évolutive ne pourrait être imputable qu'à l'hémopathie maligne, puisque ces malades n'avaient encore été soumis à aucune chimiothérapie immuno-dépressive.

- En s'aidant de l'aspect de la courbe des anticorps qui a pu être suivi chez 3 malades, on peut constater: que la parasitémie existait chez M. L... qui semblait présenter une infection récente avec ascension de la courbe des anticorps et présence d'IgM, mais qu'elle existait aussi chez $\mathbf{M}$. M... et $\mathbf{M}^{\text {me }}$ T... qui eux avaient un titre stable d'anticorps sans IgM.

Ces constatations permettent d'avancer d'une part un fait déjà bien connu : le faible rôle des anticorps dans les réactions immunologiques de défense contre le toxoplasme; d'autre part la parasitémie chez les sujets atteints de L.M.C. est sans doute une parasitémie durable, ou tout au moins des décharges parasitémiques peuvent se produire à tout moment de l'évolution. 
- L'association Spiramycine-Sulfadiazine semble avoir une action parasiticide efficace et surtout rapide: un nouvel essai d'isolement du toxoplasme 4 jours après l'institution d'un tel traitement chez $\mathbf{M}^{\mathrm{me}}$ T... s'est soldé par un échec. Il ne faut pas cependant négliger lintervention du hasard. Mais le hasard peut avoir également joué dans le résultat négatif des inoculations à partir des leucocytes des 2 autres malades: la parasitémie pouvait être absente ce jour-là ou trop minime pour être révélée à partir d'un si petit nombre d'animaux inoculés.

Tous les malades présentant une parasitémie ont été soumis à plusieurs leucophérèses, avant qu'un traitement spécifique, tant de leur toxoplasmose que de leur hémopathie, ait été institué. Nous n'avons pu retrouver que quelques dossiers cliniques de ces receveurs. La plupart avaient reçu une cure préventive de Spiramycine, dès que les résultats sérologiques des donneurs avaient été connus. Mais l'un d'entre eux, une petite fille de 3 ans, $\mathbf{M}^{11}$ B..., atteinte de leucémie aiguë lymphoblastique, a fait, de toute évidence, une toxoplasmose transfusionnelle : 8 jours après la transfusion de globules blancs sont apparus une fièvre à $39^{\circ}$ qui a persisté en plateau les jours suivants, une éruption maculaire et une séro-conversion avec un titre d'agglutinines totales au 1/4096 et d'agglutinines résistantes après action du 2 Mercaptoéthanol au 1/8. Un traitement par la Spiramycine est immédiatement institué. Les signes cliniques disparaissent en une huitaine de jours.

Très peu de temps après, l'enfant fait une « rechute méningée » puis une seconde, un mois plus tard. Elle meurt 5 mois après la découverte de sa leucose dans un état de détresse respiratoire aiguë. Aucun autre examen sérologique, ni aucun examen anatomique post-mortem n'ont été pratiqués.

D'autres observations de toxoplasmose, succédant à des transfusions de leucocytes de donneurs atteints de leucémie myéloïde chronique, ont été rapportées :

- Siegel (26) a publié 4 cas: des manifestations cliniques graves avec troubles neurologiques chez deux des quatre sujets, sont apparues 3 à 4 semaines après la transfusion, en même temps que s'établissait une conversion sérologique. Une enquête rétrospective chez les donneurs a montré que leur titre d'anticorps, qui était déjà élevé, mais sans IgM deux mois auparavant, avait encore augmenté au moment de la leucophérèse avec apparition d'IgM. Mais les essais d'isolement du toxoplasme à partir de leurs leucocytes ont été un échec.

- L'un de nous (12) a pu également évoquer, sur l'aspect de la courbe sérologique posttransfusionnelle, une toxoplasmose chez 3 receveurs de globules blancs provenant de donneurs suspects, mais l'impossibilité de suivre ces malades, cliniquement et sérologiquement suffisamment longtemps, n'a pas permis de l'affirmer.

Aussi, avec l'observation que nous venons de rapporter, c'est la première fois que la preuve réelle de transfusion de toxoplasmes a été donnée par la découverte du parasite dans le sac de leucophérèse ayant servi à transfuser le receveur. 


\section{Conclusion}

L'existence d'une sérologie toxoplasmique élevée, même stable et sans IgM, souvent discutée au cours des hémopathies malignes comme une simple réaction anamnestique sans évolutivité vraie, doit en fait correspondre dans un grand nombre de cas et, tout au moins, dans les leucémies myéloïdes chroniques à une infection toxoplasmique évolutive, même à minima, puisque dans 4 cas sur 6 nous avons pu observer une parasitémie. Il semble donc évident que les donneurs de leucocytes, atteints de L.M.C. et présentant un titre d'anticorps anti-toxoplasmiques élevé, même stable et sans IgM, soient des donneurs potentiellement dangereux. Leurs globules blancs servent en effet à transfuser des receveurs qui sont déjà dans un état critique et de moindre résistance immunitaire. Or l'on connaît la gravité que peut revêtir la greffe d'une toxoplasmose chez de tels sujets, avec dans plus de la moitié des cas une atteinte cérébrale. Il serait donc prudent d'éliminer ces sujets en tant que donneurs. $\mathrm{Si}$ cependant, pour des raisons d'urgence, une transfusion doit être faite à partir d'un donneur suspect, la gravité du risque encouru par le receveur pourrait justifier la prescription systématique chez lui d'un traitement anti-toxoplasmique sans attendre l'interprétation des résultats des examens sérologiques qui, bien sûr, seront toujours effectués.

Il est indispensable et nous nous proposons de le faire, de poursuivre les investigations et de rechercher systématiquement, par inoculation à l'animal, l'existence d'une parasitémie chez tous les donneurs L.M.C. quel que soit le titre de leurs anticorps. Il n'est pas prouvé en effet que chez de tels sujets une reviviscence kystique s'accompagne obligatoirement d'un titre d'anticorps élevé. Cette recherche systématique doit en outre comprendre une étude comparative chez les donneurs de globules blancs normaux. S'il s'avère que la parasitémie est fréquente chez les suspects atteints de leucémie myéloïde chronique, il faudra non pas les rejeter comme donneurs de globules blancs, car ils sont trop précieux mais peut-être les traiter systématiquement quelques jours avant le début des leucophérèses par la Spiramycine-Sulfadiazine, association médicamenteuse qui a montré son efficacité sur la parasitémie.

\section{Bibliographie}

Amato Neto (V.), Cotrim (J.), Laws (W. C.) et de O. Gomes (M. C.), 1963. - Nota sobre o encontro de Toxoplasma gondii em sangue destinado o transfusao. Rev. Inst. Med. Trop., Sao Paulo, 5, 68-69.

Baufine-Ducroce (H.), Couzineau (P.) et Peloux (Y.), 1973. - Agglutination directe des toxoplasmes. Feuill. biol., 14, 72, 95-97.

Berengo (A.), de lalla (F.), Cavallini (F.), Cavallina Sampieri (L.) et Bechelli (G.), 1965. - Ricerche sierologiche sulla diffusione della toxoplasmosi. Studio su 1200 donatori di sangue dell' area di Sienna. Minerva Med., 56, 4561-4570. 
Carey (R. M.), Kimball (A. C.), Armstrong (D.) et Liebermann (P. H.), 1973. - Toxoplasmosis: clinical experience in a Cancer Hospital. Am. J. Med., 54, 30-38.

COHEN (S.), 1970. - Toxoplasmosis in patients receiving immuno-suppressive therapy. $J$. Am. Med. Assoc., 211, 657-660.

Couzineau (P.), Baufine-Ducroce (H.), Peloux (Y.) et Desmonts (G.), 1973. - Le sérodiagnostic de la toxoplasmose par agglutination directe. Nouv. Presse Méd., 2, 1604-1606.

Desmonts (G.), Le Tan Vinh et Cousin (L.), 1957. - L'isolement du toxoplasme par inoculation à l'animal. Rev. Fr. Clin. Biol., 2, 555-565.

Desmonts (G.), 1960. - Diagnostic sérologique de la toxoplasmose. Path. Biol., 8, 109-125.

Desmonts (G.), Couvreur (J.), Colin (J.), Peupiou (J.), 1972. - Vers un diagnostic précoce de la toxoplasmose aiguë. Etude critique du test de Remington. Nouv. Presse Méd., $1,339-342$.

DuKA (T. V.), 1966. - Toxoplasmose et infestation des donneurs par toxoplasme. Klin. Med. (Moscou), 44, 127-130.

FrenKel (J. K.), 1957. - Effect of Cortisone, total body radiation and Nitrogen mustard on chronic latent Toxoplasmosis. Amer. J. Path., 33, 618-619.

GARIN (Y.), 1973. - I. La réaction d'agglutination directe des toxoplasmes. II. Application à l'étude de la toxoplasmose au cours des transfusions sanguines. Thèse médecine, Paris.

Giraud (H.), Laugier $\left(\mathbf{M}^{\mathrm{me}}\right)$ et Maestrali $\left(\mathbf{M}^{11 \mathrm{e}}\right)$, 1961. - Premiers résultats d'une étude sur la parasitémie dans la toxoplasmose expérimentale. Arch. Fr. Pédiat., 18, 1261-68.

JACOBS (L.) et JoNes (F. E.), 1950. - The parasitemia in experimental toxoplasmosis. J. Infect. Dis., 87, 78.

JAcobs (L.), 1973. - New knowledge of toxoplasma and toxoplasmosis. Adv. Parasitol., 631-669.

Kimball (A. C.), Kean (B. U.) and Kellner (A. R.), 1965. - The risk of transmitting Toxoplasmosis by blood transfusion. Transfusion, 5, 447-451.

Lunde (M. N.), Gelderman (A. H.), Hayes (S. L.) and Vogel (C. L.), 1970. - Serologic diagnosis of active toxoplasmosis complicating malignant disease. Cancer, 25, 3, 637-643.

Miller (M. J.), Aronson (W. J.), Remington (J. S.), 1966. - Persistant parasitemia in human toxoplasmosis (abstract). Clin. Res., 14, 145.

Miller (M. J.), Aronson (W. J.), Remington (J. S.), 1969. - Late parasitemia in asymptomatic acquired toxoplasmosis. Ann. Intern. Med., 71, 135-145.

NeU (H. C.), 1972. - Toxoplasmose: Complication des affections malignes. Rev. Med., 9, 487-490.

Prior (J. A.), Cole (C.), Sarlow (S.), Chamberlain (D. M.), 1953. - Toxoplasmosis. IV. Report of three cases with particular reference to asymptomatic toxoplasma parasitemia in a young woman. Arch. Int. Med. (Chicago), 92, 314.

Remington (J. S.), Melton (M. L.), Jacobs (L.), 1961. - Induced and spontaneous recurrent parasitemia in chronic infection with avirulent strain of Toxoplasma gondii. J. Immun., 87, 578. 
Roth (J. A.), Siegel (S.), Levine (A.) and Berard (G.), 1971. - Fatal recurrent toxoplasmosis in a patient initially infected with a leukocyte transfusion. Amer. J. Clin. Path., 56, 601-605.

Sibalic (D.), 1975. - A study of parasitemia in patients with acute and chronic Toxoplasmosis. Acta Parasitol. Iugoslavica, 6, 7-11.

Sibalic (D.), Rodovic (M.), Martinovic (H.), 1975. - Isolation of Toxoplasma gondii from human blood and the survival of the parasites in the blood in vitro. Acta Parasitol. Iugoslavica, 6, 13-18.

Siegel (S. E.), Lunde (M. N.), Gelderman (A. H.), Halterman (R. H.), Brown (J. A.), Levine (A. S.) and Graw (R. G.) Jr., 1971. - Transmission of toxoplasmosis by leukocyte transfusion. Blood., 37, 388-394.

VIETZKE (W. M.) et al., 1968. - Toxoplasmosis complicating malignancy. Cancer, 21, 816-827.

Vogel (C. C.) and Lunde (M. P.), 1969. - Toxoplasma serology in patients with malignant diseases of the reticuloendothelial system. Cancer, 23, 614-618. 